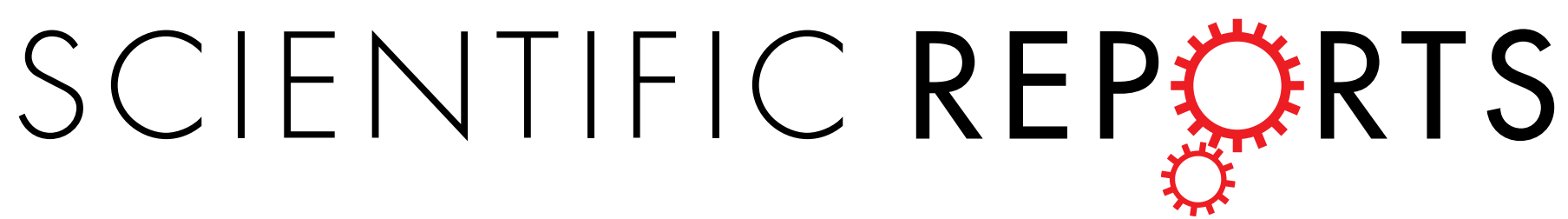

\title{
OPEN Genetic-deletion of
}

\section{Cyclooxygenase-2 Downstream} Prostacyclin Synthase Suppresses

Received: 08 September 2015

Accepted: 29 October 2015

Published: 27 November 2015 \section{Inflammatory Reactions but} Facilitates Carcinogenesis, unlike Deletion of Microsomal Prostaglandin E Synthase-1

Yuka Sasaki $^{1}$, Shuhei Kamiyama ${ }^{1}$, Azusa Kamiyama ${ }^{1}$, Konomi Matsumoto ${ }^{1}$, Moe Akatsu ${ }^{1}$, Yoshihito Nakatani ${ }^{1}$, Hiroshi Kuwata ${ }^{1}$, Yukio Ishikawa ${ }^{2}$, Toshiharu Ishii ${ }^{3}$, Chieko Yokoyama ${ }^{4}$ \& Shuntaro Hara ${ }^{1}$

Prostacyclin synthase (PGIS) and microsomal prostaglandin E synthase-1 (mPGES-1) are prostaglandin (PG) terminal synthases that function downstream of inducible cyclooxygenase (COX)- 2 in the $\mathrm{PGI}_{2}$ and $\mathrm{PGE}_{2}$ biosynthetic pathways, respectively. mPGES-1 has been shown to be involved in various COX-2-related diseases such as inflammatory diseases and cancers, but it is not yet known how PGIS is involved in these COX-2-related diseases. Here, to clarify the pathophysiological role of PGIS, we investigated the phenotypes of PGIS and mPGES-1 individual knockout (KO) or double KO (DKO) mice. The results indicate that a thioglycollate-induced exudation of leukocytes into the peritoneal cavity was suppressed by the genetic-deletion of PGIS. In the PGIS KO mice, lipopolysaccharide-primed pain nociception (as assessed by the acetic acid-induced writhing reaction) was also reduced. Both of these reactions were suppressed more effectively in the PGIS/ mPGES-1 DKO mice than in the PGIS KO mice. On the other hand, unlike mPGES-1 deficiency (which suppressed azoxymethane-induced colon carcinogenesis), PGIS deficiency up-regulated both aberrant crypt foci formation at the early stage of carcinogenesis and polyp formation at the late stage. These results indicate that PGIS and MPGES-1 cooperatively exacerbate inflammatory reactions but have opposing effects on carcinogenesis, and that PGIS-derived $\mathrm{PGI}_{2}$ has anti-carcinogenic effects.

Non-steroidal anti-inflammatory drugs (NSAIDs) exert their anti-inflammatory and anti-tumor effects by reducing the production of prostaglandins (PGs) by inhibiting cyclooxygenase (COX) ${ }^{1-3}$. Of the two COX isozymes COX-1 and COX-2, COX-1 is expressed constitutively in most tissues and is generally

${ }^{1}$ Division of Health Chemistry, Department of Healthcare and Regulatory Sciences, School of Pharmacy, Showa University, Tokyo 142-8555, Japan. ${ }^{2}$ Pathology Section, Itabashi Medical Laboratory, Tokyo 174-0051, Japan. ${ }^{3}$ Department of Pathology, Saiseikai Yokohama City Tobu Hospital, Yokohama 230-8765, Japan. ${ }^{4}$ Kanagawa Institute of Technology, Atsugi 243-0292, Japan. Correspondence and requests for materials should be addressed to S.H. (email: haras@pharm.showa-u.ac.jp) 
responsible for the production of the PGs that control normal physiological functions, whereas COX-2 is induced in response to mitogens, cytokines, and cellular transformation and is related to inflammatory reactions and carcinogenesis. The long-term use of NSAIDs is associated with severe side effects, mainly gastrointestinal injury and renal irritations, apparently due to impaired COX-1-dependent PG biosynthesis. Although COX-2-selective inhibitors show reduced gastrointestinal complications, recent clinical trials indicated a significantly increased cardiovascular risk of these agents ${ }^{4}$. The specific inhibition of COX-2 alters the balance between platelet-derived thromboxane $\mathrm{A}_{2}\left(\mathrm{TXA}_{2}\right)$ and endothelium-derived prostacyclin $\left(\mathrm{PGI}_{2}\right)$, leading to increases in the risk of thrombosis due to altered vascular tone. Thus, for the development of novel NSAIDs without adverse side effects, a more selective modulation of PG production appears to be desirable.

$\mathrm{PGH}_{2}$, a COX metabolite, is converted to each PG species by species-specific PG terminal synthases. Among the PG terminal synthases, microsomal PGE synthase-1 (mPGES-1) is induced by pro-inflammatory stimuli or mitogens and down-regulated by anti-inflammatory glucocorticoids, as in the case of COX-2, and is functionally coupled with COX-2 in marked preference to COX-1 ${ }^{5-8}$. The induction of mPGES-1 expression has been observed in various conditions and processes in which COX2-driven $\mathrm{PGE}_{2}$ has been implicated, including rheumatoid arthritis and cancer. Other research groups and our group have used mPGES-1 knockout (KO) mice, finding that mPGES-1 plays a critical role in inflammatory reactions and carcinogenesis ${ }^{7-11}$. Now, mPGES-1 has gained attention as a novel target for NSAIDs. Several mPGES-1-selective inhibitors were recently developed and shown to suppress inflammatory reactions in experimental animal models ${ }^{12-14}$.

However, not only mPGES-1 but also PGI 2 synthase (PGIS) functionally couples with COX-25,15. In mPGES-1 KO mice, COX-2-derived $\mathrm{PGH}_{2}$ is metabolically shunted into PGIS-mediated $\mathrm{PGI}_{2}$ production $^{9,11,16}$. PGIS is highly expressed in vascular endothelial and smooth muscle cells, but a variety of cells other than vascular cells including macrophages also express PGIS ${ }^{17-20}$. Studies using $\mathrm{PGI}_{2}$ receptor (IP)-KO mice have also revealed that $\mathrm{PGI}_{2}$ is involved in inflammatory and pain responses as well as the regulation of vascular tone $\mathrm{e}^{21-24}$, but the question of how PGIS is involved in COX-2-related diseases such as inflammatory diseases and cancers has not been fully answered.

To reveal the pathophysiological roles of PGIS in inflammatory reaction and carcinogenesis, we here established PGIS and mPGES-1 double knockout (DKO) mice and investigated the phenotypes of PGISand mPGES-1-single KO mice and the DKO mice. The results demonstrated that the genetic-deletion of PGIS suppresses inflammatory reactions but facilitates carcinogenesis, unlike mPGES-1 deletion. These findings indicated that understanding the relationships among the PG terminal synthases is crucial to the development of novel NSAIDs without adverse side effects.

\section{Results}

Establishment of PGIS/mPGES-1 DKO mice and characterization of macrophages derived from KO mice. For the establishment of PGIS $(-/-)$ mPGES-1 $(-/-)$ mice (DKO mice), PGIS $(-/-)$ mice were crossed with mPGES-1 $(-/-)$ mice to generate PGIS $(+/-)$ mPGES-1 $(+/-)$ mice, and then these heterozygous mice were intercrossed. In addition, PGIS $(-/-)$ mPGES-1 $(-/-)$ mice were crossed with PGIS(+/-)mPGES-1 (+/-) mice. Among the 233 progenies of these crosses, $73(31.3 \%)$ were PGIS $(+/-)$ mPGES-1(+/-) (as control mice in the following study), 59 (25.3\%) were PGIS(-/-) mPGES-1 $(+/-)$ (as PGIS KO mice), 55 (23.6\%) were PGIS $(+/-)$ mPGES-1 $(-/-)$ (as mPGES-1 KO mice), and 46 (19.7\%) were PGIS(-/-)mPGES-1(-/-) (as PGIS/mPGES-1 DKO mice). The number of DKO mice was slightly less than the expected Mendelian ratio, but all four genotypes of mice were born.

For the determination of whether PGIS and mPGES-1 were indeed knocked out in the null mice, the protein levels in thioglycollate-induced peritoneal macrophages $(M \Phi s)$ prepared from the four genotypes of mice were analyzed by Western blotting (Fig. 1A). In the control and PGIS-deficient M $\Phi$ s, mPGES-1 protein was detected in normal culture conditions as was COX-2, and mPGES-1 was up-regulated by the lipopolysaccharide (LPS) treatment. On the other hand, PGIS protein was detected in the control

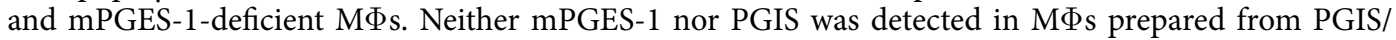
mPGES-1 DKO mice. Since it has been reported that LPS-stimulated PGE 2 production was markedly suppressed and the production of other prostanoids was increased in M $\Phi$ s derived from mPGES-1 KO mice relative to those derived from wild-type (WT) mice $e^{9,16,25,26}$, we further measured prostanoids in culture medium from four genotypes of $M \Phi$ s. As reported previously, mPGES-1 deficiency decreased the $\mathrm{PGE}_{2}$ levels but conversely increased the levels of PGs other than $\mathrm{PGE}_{2}$ including 6-ketoPGF ${ }_{1 \alpha}$, a $\mathrm{PGI}_{2}$ metabolite, in culture medium (Fig. 1B), indicating that COX-2-derived $\mathrm{PGH}_{2}$ is metabolically shunted into the other prostanoid synthetic pathway in mPGES-1-deficient M $\Phi$ s.

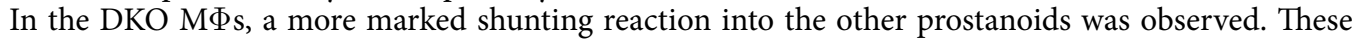
shunting phenomena were not observed in PGIS-deficient $M \Phi s$, in whose culture medium the levels of prostanoids including $\mathrm{PGE}_{2}$ were similar to those in the control $\mathrm{M} \Phi \mathrm{s}$. These results suggested that the intracellular shunting of COX-2-derived $\mathrm{PGH}_{2}$ might have a single direction in mouse peritoneal $\mathrm{M} \Phi$ s. In M $\Phi$, mPGES-1 might be not able to metabolize $\mathrm{PGH}_{2}$, which should be supplied to PGIS from COX-2.

It has been shown that PGIS and mPGES-1 are expressed in mouse kidney and lung even under basal conditions $9,16,18,19,27$. We next measured the prostanoid levels in kidneys and lungs prepared from the four genotypes of mice and found that these two organs also showed a shunting reaction similar to that of 

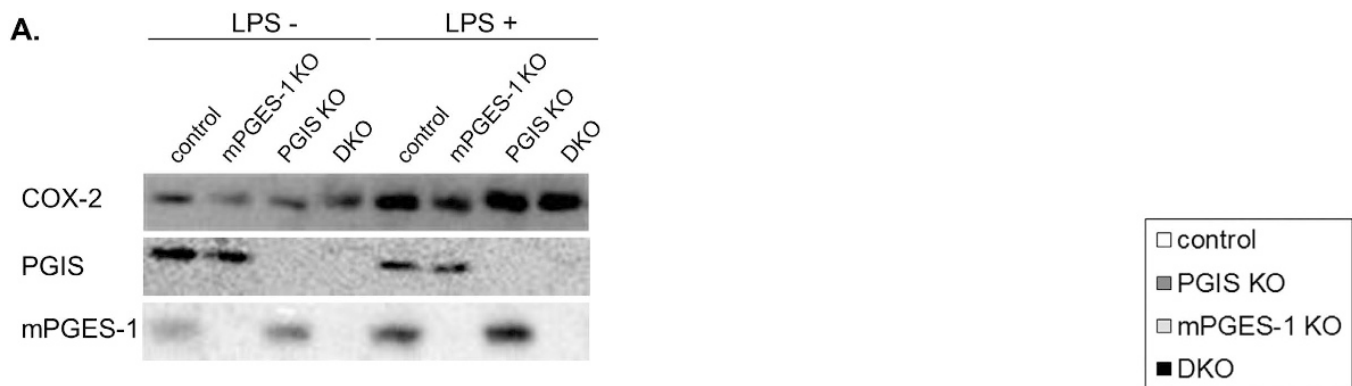

B $\mathrm{PGE}_{2}$
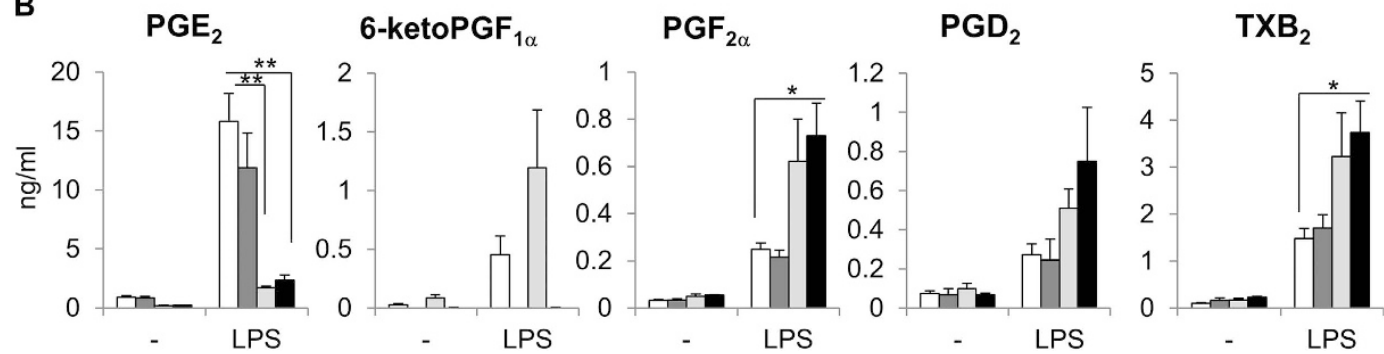

Figure 1. Expression of COX-2, PGIS and mPGES-1 and production of prostanoids in peritoneal MФs prepared from PGIS and/or mPGES-1 KO mice. (A) Immunoblot analysis of COX-2, PGIS and mPGES-1 expression in the control, mPGES-1 KO, PGIS KO, and PGIS/mPGES-1 DKO M $\Phi$ s. Peritoneal M $\Phi$ s were prepared from thioglycollate-treated mice, and then incubated with or without LPS for 24 hours. Cell lysates were prepared and subjected to immunoblot analysis. (B) Amounts of prostanoids in culture medium from these $M \Phi$ s. Culture media from LPS-treated M $\Phi$ s were subjected to a lipidomics analysis using LC-ESI-MS. Results are mean $\pm \operatorname{SEM}(\mathrm{n}=3-8) .{ }^{\star} \mathrm{P}<0.05$ vs. control.

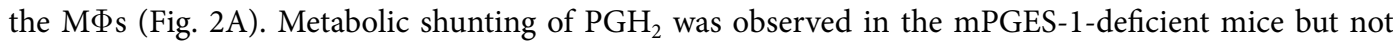
in PGIS-deficient mice. We further found that the DKO mice developed renal disorders with arterial sclerosis and hypertrophy of vessels (Fig. 2B), as did the PGIS single-KO mice ${ }^{28}$. These results suggested that renal disorders observed in PGIS-deficient mice might be induced by a breakdown of $\mathrm{PGI}_{2}$ levels but not by shunting into prostanoids other than $\mathrm{PGI}_{2}$.

PGIS is involved in inflammatory reactions. Studies using KO mice have revealed that IP and $\mathrm{PGE}_{2}$ receptor subtypes EP2 and EP4 are involved in inflammatory reactions including swelling and pain $^{21-24}$. Thus, to examine the effects of PGIS and mPGES-1 deficiency on inflammatory reaction, we next counted the number of exudate leukocytes and measured the levels of prostanoids in the peritoneal fluids prepared from the four genotypes of thioglycollate-treated mice. As shown in Fig. 3, in the control mice, the peritoneal leukocyte number was increased steadily from day 2 to 4 after the injection of thioglycollate, accompanied by an increase in 6-ketoPGF ${ }_{1 \alpha}$ levels. PGIS deficiency did not significantly affect the number of exudate leukocytes on day 2, but significantly decreased their number on day 4 compared to the control mice. Our morphological analysis revealed that among leukocytes, the

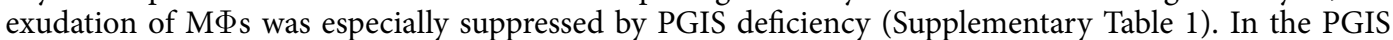

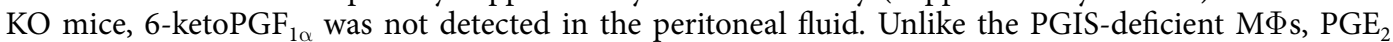
and the other prostanoid levels in the PGIS KO mice were higher than those in the control mice. These results indicated that PGIS-derived $\mathrm{PGI}_{2}$ might play an important role in the exudation of $M \Phi s$ at the late stage of inflammatory reaction.

Moreover, the migration of M $\Phi$ s into the peritoneal fluid was not affected by mPGES-1 deficiency but was suppressed more effectively in the PGIS/mPGES-1 DKO mice than in the PGIS KO mice. mPGES1-derived $\mathrm{PGE}_{2}$ might contribute to the exudation of $\mathrm{M} \Phi \mathrm{s}$, but its contribution might be smaller than that of PGIS-derived $\mathrm{PGI}_{2}$.

We further investigated the effect of PGIS deficiency on inflammatory pain hypersensitivity, as assessed by the LPS-primed acetic acid-induced writhing reaction. The LPS pretreatment induced the expressions of COX-2 and mPGES-1 and then enhanced the writhing reaction. As we previously reported ${ }^{9}$, the injection of acetic acid into the peritoneum of mice induced a stretching behavior, that peaked at 5-10 min and then declined gradually over $30 \mathrm{~min}$. The writhing reaction was reduced in both the PGIS KO and mPGES-1 KO mice, and it was suppressed more effectively in the PGIS/mPGES-1 DKO mice compared to the PGIS KO and mPGES-1 KO mice (Fig. 4A). In the PGIS KO mice, 6-ketoPGF ${ }_{1 \alpha}$ was not observed but the $\mathrm{PGE}_{2}$ levels were markedly increased compared to the control mice, although a similar shunting phenomenon was not observed in the mPGES-1 KO mice (Fig. 4B). In the PGIS/mPGES-1 DKO mice, 
A

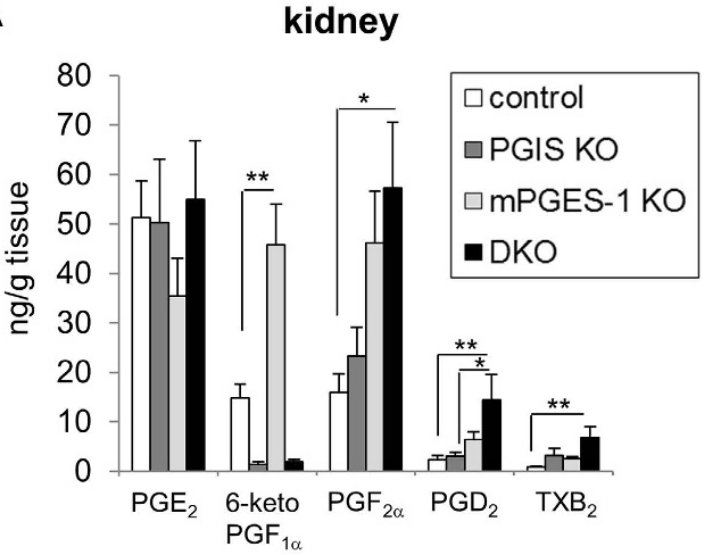

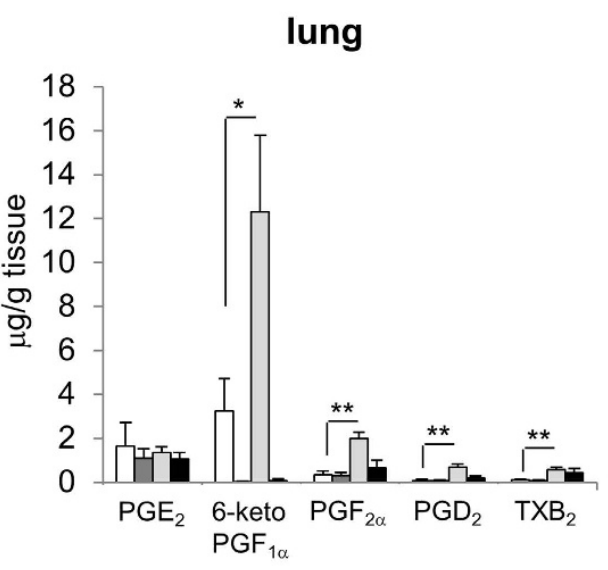

B
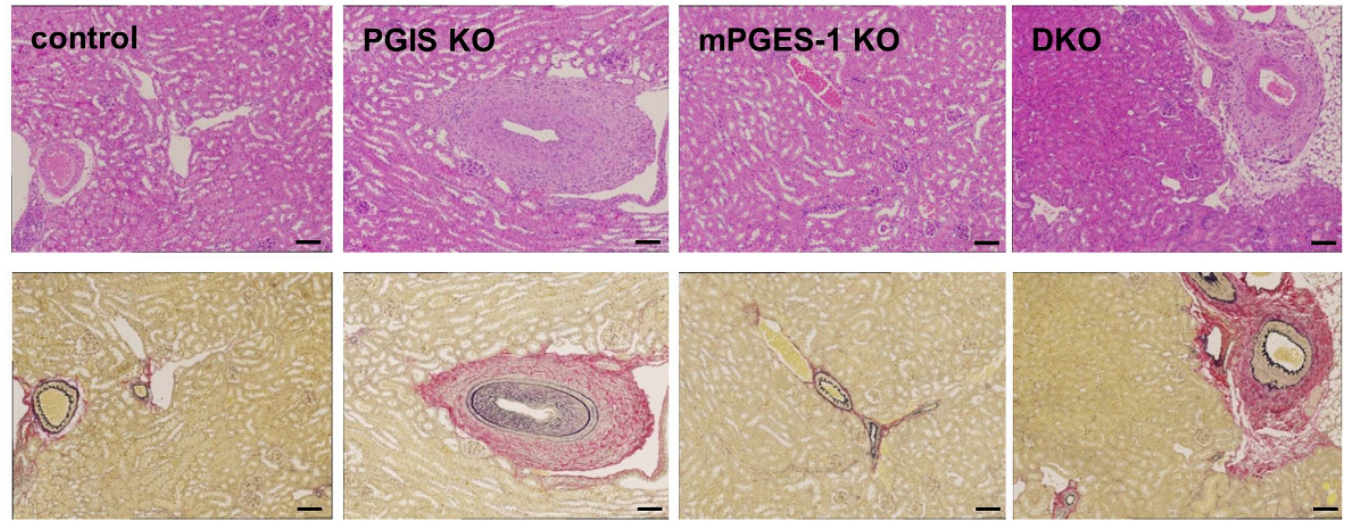

Figure 2. Prostanoid levels in kidney and lungs and histological features of kidney prepared from PGIS and/or mPGES-1 KO mice. (A) Prostanoid levels in kidneys and lungs. Kidneys and lungs were resected from mice and then homogenized. Their lipids were extracted and subjected to a lipidomics analysis by LCESI-MS. Results are mean \pm SEM $(\mathrm{n}=3-6) .{ }^{*} P<0.05$ and ${ }^{* *} P<0.01$ vs. control. (B) Histological features of kidneys. Sections of kidneys were stained with hematoxylin and eosin (H\&E, upper panel) or Elastica van Gieson stain (lower panel). Bar: $100 \mu \mathrm{m}$.

the levels of both 6-ketoPGF ${ }_{1 \alpha}$ and $\mathrm{PGE}_{2}$ were reduced. These results indicate that PGIS-derived $\mathrm{PGI}_{2}$ facilitates inflammatory pain hypersensitivity in a coordinated manner with mPGES-1-derived PGE $_{2}$.

PGIS is involved in chemically-induced carcinogenesis. We next injected azoxymethane (AOM) intraperitoneally into these four genotypes of mice once a week for 6 weeks to induce colon carcinogenesis. To examine the involvement of PGIS in an early phase of carcinogenesis, we first killed animals 6 weeks after the last injection, and evaluated the preneoplastic aberrant crypt foci (ACF) formation (Fig. 5A). The results indicated that PGIS deficiency did not induce spontaneous colon carcinogenesis but significantly increased the number of ACF, whereas mPGES-1 deficiency decreased the ACF number, as described previously ${ }^{11}$. In the PGIS/mPGES-1 DKO mice, the ACF number was similar to that in the control mice. These results indicate that the genetic-deletion of PGIS facilitates tumor propagation even though it is not sufficient for tumor initiation.

At 20 weeks after the last injection, the AOM administration induced the development of multiple tumors in the control colons, whereas mPGES-1 deficiency suppressed the colon carcinogenesis as described previously ${ }^{11}$. In contrast to mPGES-1 deficiency, PGIS deficiency tended to increase the polyp numbers (Fig. 5B). In addition, the number of large polyps was significantly increased in the PGIS KO mice. As shown in Fig. 5C, histologically, adenocarcinomas were observed in the control, PGIS KO and DKO mice, but only adenomas were observed in the mPGES-1 KO mice. Among them, the adenocarcinomas in the PGIS KO mice were considerably larger than those in the control mice. Balb/c background mice were used for these analyses, because it was shown that $\mathrm{Balb} / \mathrm{c}$ mice are more sensitive to AOM-induced colon carcinogenesis than other strain mice $^{29}$. We also found that the number of colon polyps (and the number of large polyps in particular) was increased in the PGIS KO mice compared to the wild type (WT) mice even in the C57BL/6 mouse strain, which has been well characterized as being highly resistant to colon tumor induction by $\mathrm{AOM}^{29}$ (Fig. 5D). These results indicated that the genetic-deletion of PGIS exacerbates chemically induced colon carcinogenesis. 
A

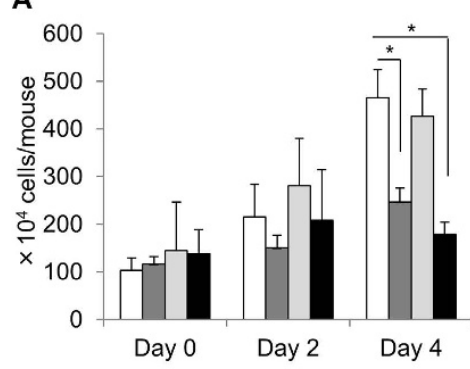

C

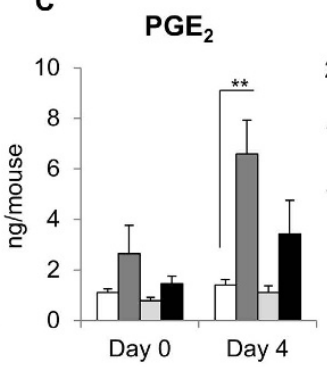

6-ketoPGF $1 \alpha$

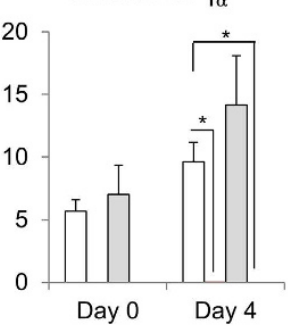

$\mathrm{PGD}_{2}$

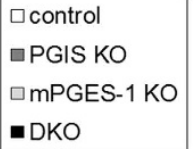

- DKO
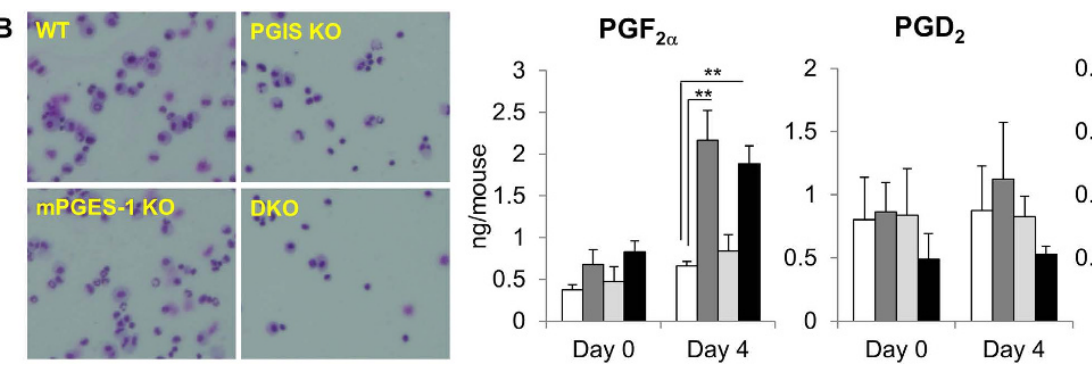

$\mathrm{TXB}_{2}$

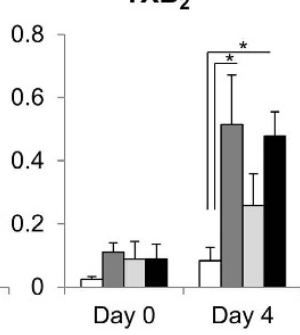

Figure 3. Involvement of PGIS and mPGES-1 in thioglycollate-induced peritonitis. Number and morphology of exudate leukocytes $(\mathbf{A}, \mathbf{B})$ and prostanoid levels in peritoneal fluids $(\mathbf{C})$ from thioglycollatetreated PGIS and/or mPGES-1 KO mice. (A) Peritoneal exudate cells and fluids were collected from mice on day 0,2 or 4 after the injection of thioglycollate. The peritoneal cells were washed and then counted.

(B) Representative Giemsa staining of cytocentrifuge preparations of the exudate leukocytes on day 4 .

(C) The peritoneal fluids were subjected to a lipidomics analysis by LC-ESI-MS. Results are mean \pm SEM $(\mathrm{n}=3-6) .{ }^{*} P<0.05$ and ${ }^{*} P<0.01$ vs. control.

A

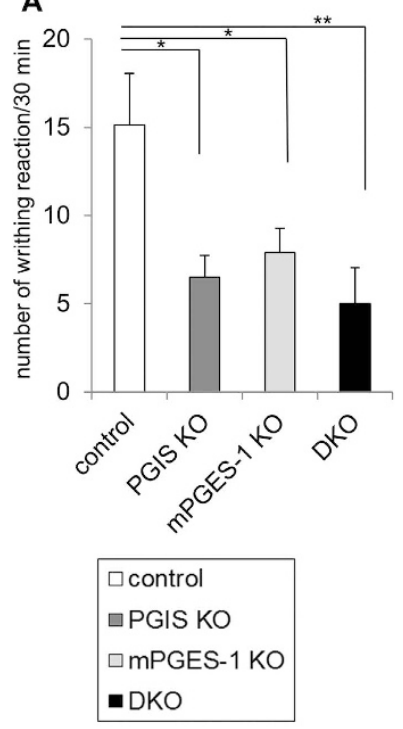

B
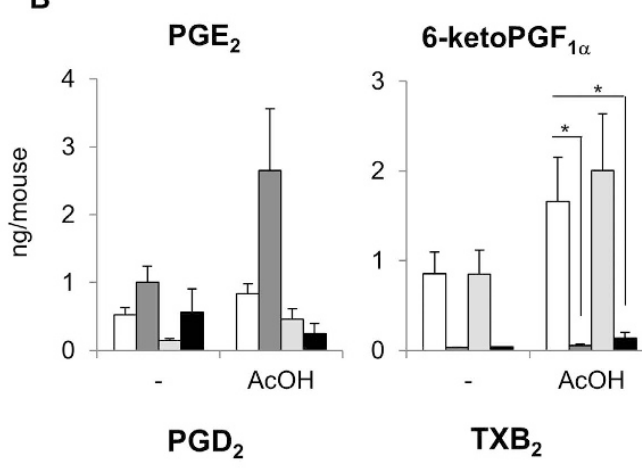

$\mathrm{PGF}_{2 \alpha}$

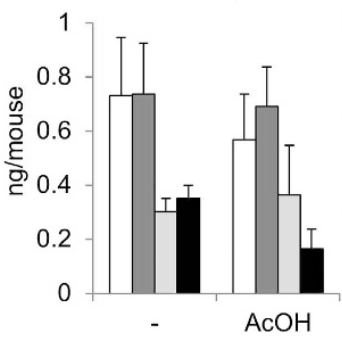

$\mathrm{TXB}_{2}$

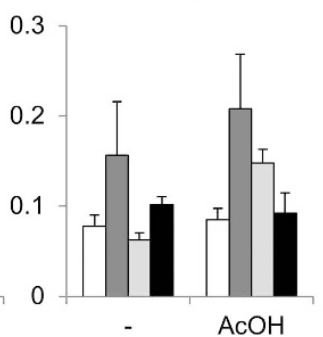

Figure 4. Involvement of PGIS and mPGES-1 in the LPS-primed acetic acid-induced writhing reaction. Number of writhing reactions (A) and prostanoid levels in peritoneal fluids (B) from LPS-primed acetic acid-treated PGIS and/or mPGES-1 KO mice. (A) The writhing reaction was induced in mice by an injection of acetic acid 18 hours after LPS treatment. The number of writhing responses was counted for $30 \mathrm{~min}$ after the injection of acetic acid. (B) Peritoneal fluids were collected from mice $10 \mathrm{~min}$ after the injection of acetic acid $(+)$ or saline $(-)$ and then subjected to a lipidomics analysis by LC-ESI-MS. Results are mean \pm SEM $(\mathrm{n}=6-10) .{ }^{\star} P<0.05$ and ${ }^{*} P<0.01$ vs. control. 
A

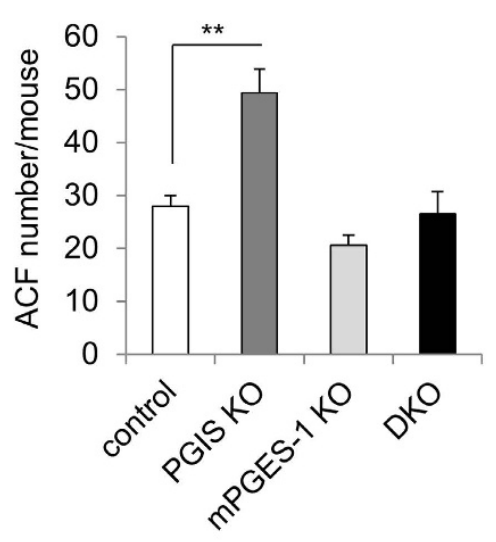

C
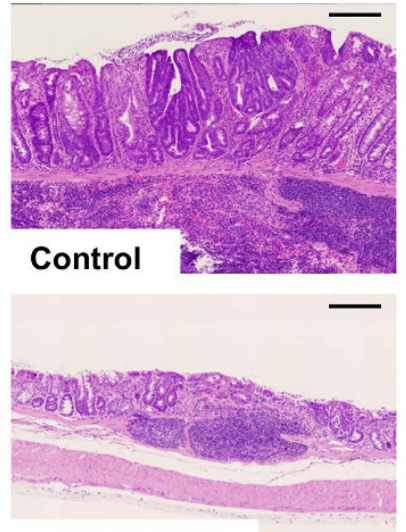

MPGES-1 KO
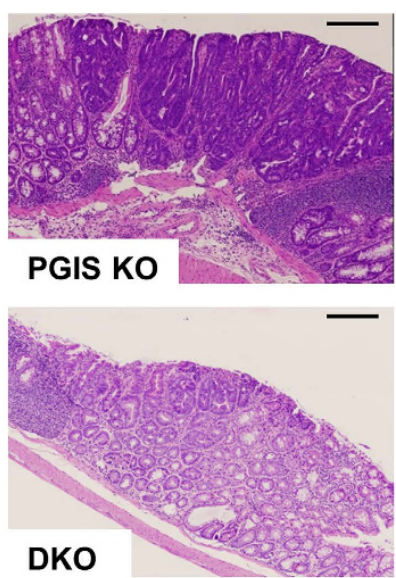

B

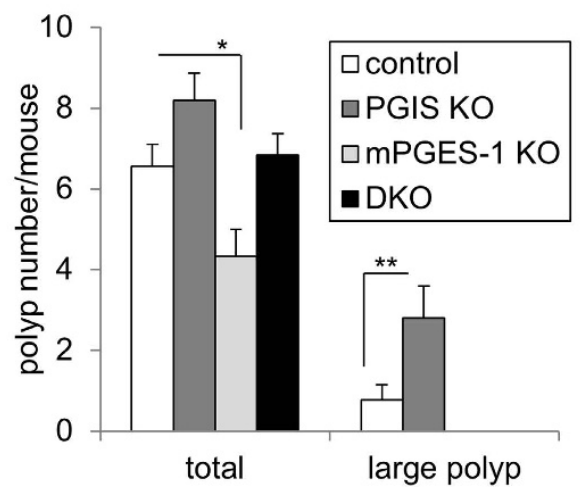

D

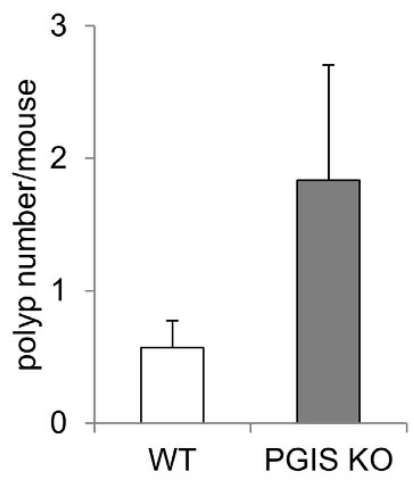

Figure 5. Involvement of PGIS and mPGES-1 in AOM-induced colon carcinogenesis. Numbers of ACFs (A) and polyps (B,D) in the colon tissues from AOM-treated PGIS and/or mPGES-1 KO mice. (A) PGIS and/or mPGES-1 KO mice on a Balb/c background were intraperitoneally injected with AOM once a week for 6 weeks and killed 6 weeks after the last injection. The number of ACFs per mouse in the colon tissues is shown. Results are mean $\pm \operatorname{SEM}(\mathrm{n}=4-5)$. ${ }^{* *} P<0.01$ vs. control. (B) PGIS and/or mPGES-1 KO mice on a Balb/c background were killed 20 weeks after the last injection of AOM. The numbers of total polyps and large $(>2 \mathrm{~mm})$ polyps per mouse in the colon tissues are shown. Results are mean \pm SEM $(\mathrm{n}=5-9)$. ${ }^{\star} P<0.05$ and ${ }^{\star *} P<0.01$ vs. control. (C) Histological features of colon tissues. Representative H\&E staining of the colon tissues from AOM-treated mice. Bar: $100 \mu \mathrm{m}$. (D) PGIS KO and littermate WT mice on a C57BL/6 background were intraperitoneally injected with AOM and killed 20 weeks after the last injection. The numbers of total polyps and large $(>2 \mathrm{~mm})$ polyps per mouse in the colon tissues are shown. Results are mean $\pm \operatorname{SEM}(\mathrm{n}=6-9) .{ }^{\star} P<0.05$ and ${ }^{\star *} P<0.01$ vs. control.

We next analyzed the levels of prostanoids in these colon polyp tissues (Fig. 6A). As expected, in polyp tissues, mPGES-1 deficiency decreased the PGE $_{2}$ levels, and 6-ketoPGF ${ }_{1 \alpha}$ was not detected in the PGIS KO mice or DKO mice. The levels of PGs other than $\mathrm{PGE}_{2}$ including 6-ketoPGF $\mathrm{P}_{1 \alpha}$ in the polyp tissues of the mPGES-1 KO mice were higher than those in the control mice, but a similar shunting phenomenon was not observed in the colon polyp tissues of the PGIS KO mice. In the PGIS KO mice, the level of $\mathrm{PGE}_{2}$ was similar to that in the control mice. We analyzed the expression levels of COX-2, PGIS and mPGES-1 in colon tissues by quantitative RT-PCR. As shown in Fig. 6B, the levels of both COX-2 and mPGES-1 mRNA in polyp tissues were substantially higher than those in normal tissues of the colon. On the other hand, the PGIS mRNA level in the polyps was similar to that in the normal tissues. In our immunohistochemical analysis, the positive immunostaining signal of PGIS was observed only in blood vessels, not in tumor cells or tumor stromal cells (Fig. 6C). These results suggested that PGIS in host-derived vascular cells might be involved in carcinogenesis and that the breakdown of $\mathrm{PGI}_{2}$ in host-derived vascular cells might lead to an exacerbation of colon tumors in PGIS KO mice.

\section{Discussion}

We here established mice that are doubly deficient for PGIS and mPGES-1, both of which are preferentially coupled with COX-2 as their upstream enzymes, and we then investigated the phenotypes of these mice. PGIS/mPGES-1 DKO mice were born. However as well as COX-2 KO mice ${ }^{30}$, when heterozygous $\mathrm{KO}$ mice were intercrossed, the number of DKO mice was slightly less than the expected Mendelian 


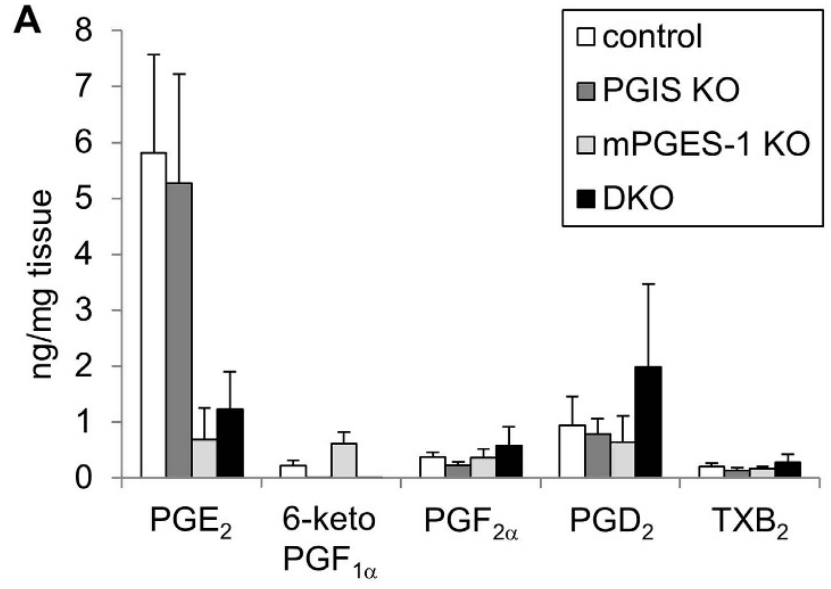

B

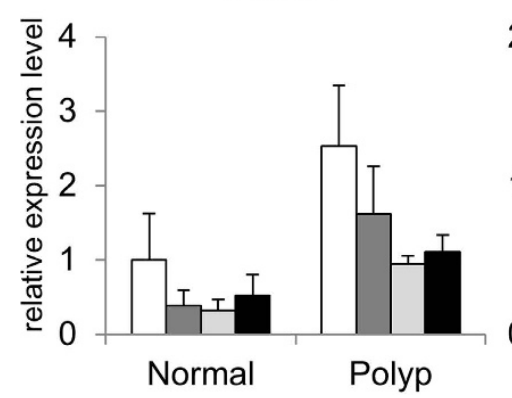

PGIS

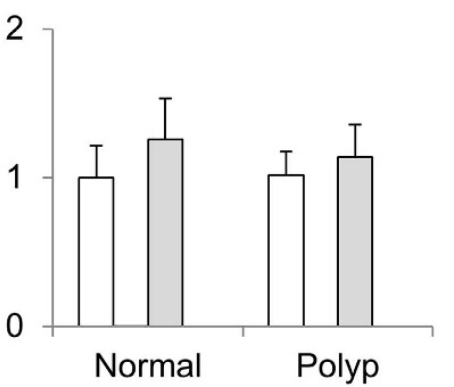

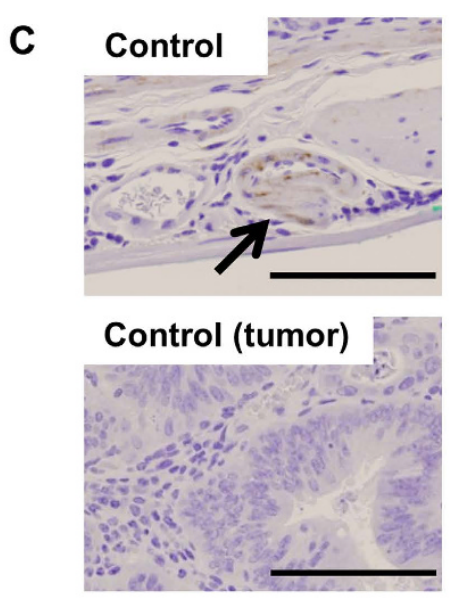

MPGES-1

Figure 6. Prostanoid levels and expression of COX-2, PGIS and mPGES-1 in colon tissues prepared from AOM-treated PGIS and/or mPGES-1 KO mice. PGIS and/or mPGES-1 KO mice on a Balb/c background were intraperitoneally injected with AOM once a week for 6 weeks and killed 20 weeks after the last injection. Tissues from each colon tissue were divided into normal and tumor tissues. (A) Prostanoid levels in colon tumor tissues. Lipids were extracted from colon tumor tissues of AOM-treated mice and subjected to a lipidomics analysis by LC-ESI-MS. Results are mean \pm SEM $(n=4-7)$. (B) Expression of COX-2, PGIS and mPGES-1 mRNAs in colon tissues. COX-2, PGIS and mPGES-1 mRNA levels in normal or tumor colon tissues from AOM-treated mice were assessed by real-time RT-PCR. Results are mean \pm SEM $(\mathrm{n}=3-5)$. (C) Representative PGIS-immunostaining of the normal or tumor colon tissues from AOMtreated WT mice. Bar: $100 \mu \mathrm{m}$.

ratio. We also observed the development of renal disorders in the DKO mice (Fig. $2 \mathrm{~B}$ ) as well as in the COX-2 $\mathrm{KO}^{30}$ and PGIS KO mice ${ }^{28}$. These results suggested that renal disorders might be induced by a breakdown of COX-2/PGIS-derived $\mathrm{PGI}_{2}$ levels.

The inflammatory reactions and inflammatory pain hypersensitivity were significantly suppressed in the PGIS KO mice, and they tended to be suppressed more effectively in the PGIS/mPGES-1 DKO mice than in the PGIS KO mice (Figs 3A and 4A). It has been shown that IP as well as EP receptors and mPGES-1 are involved in inflammatory reactions including swelling and pain ${ }^{21-24}$. The present results indicated that PGIS-derived $\mathrm{PGI}_{2}$ acts on IP at inflamed sites and exacerbates inflammation together with mPGES-1-derived $\mathrm{PGE}_{2}$. It is noteworthy that PGIS deficiency increased the $\mathrm{PGE}_{2}$ levels in inflammatory exudates but mPGES-1 deficiency did not affect the production of PGs other than $\mathrm{PGE}_{2}$ in inflammatory exudates (Figs $3 \mathrm{~B}$ and $4 \mathrm{~B}$ ).

The shunting pattern of $\mathrm{PGH}_{2}$ observed in the exudates from $\mathrm{KO}$ mice was different from that in

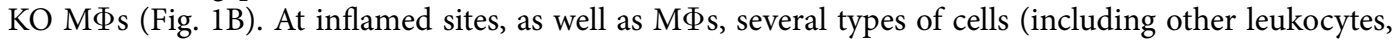
vascular cells and stromal cells) are able to produce PGs. In the present case, M $\Phi$ s might contribute less to PG production. Otherwise, the intracellular transport of $\mathrm{PGH}_{2}$ from PGIS-deficient $\mathrm{M} \Phi$ s to the other inflammatory cells might occur. For the development of inhibitors specific for each PG terminal synthase as anti-inflammatory drugs, it is necessary to determine which types of cells are involved in the process of the target illnesses. Here, we found that mPGES-1 deficiency suppressed the acetic acid-induced pain response but did not affect the thioglycollate-induced leukocyte exudation (Figs 3A and 4A). Unlike COX-2, mPGES-1 might have only limited involvement in certain types of inflammatory reactions.

As shown in Fig. 5, the deletions of PGIS and mPGES-1 showed opposite effects on the chemically-induced colon carcinogenesis. mPGES-1 deficiency suppressed the AOM-induced ACF 
and polyp formation, but PGIS deficiency enhanced both of them. Keith et al. reported that in a smoke-exposure model, pulmonary-specific PGIS-overexpressing mice were chemoprotected from developing lung tumors ${ }^{31}$. Together these findings indicate that PGIS-derived $\mathrm{PGI}_{2}$ functions as an anti-carcinogenic agent in several types of cancers. In colon tumor tissues, PGIS was expressed in blood vessels (Fig. 6C) and mPGES-1 was expressed in tumor cells and tumor stromal cells ${ }^{11}$. When tumor and host-associated COX-2/mPGES-1-derived $\mathrm{PGE}_{2}$ increase and then exceed the anti-carcinogenic actions of vascular COX-2/PGIS-derived $\mathrm{PGI}_{2}$, a tumor might begin to progress. As described previously ${ }^{11}$, the ablation of mPGES-1 resulted not only in the suppression of carcinogenic $\mathrm{PGE}_{2}$ production but also in the enhancement of anti-carcinogenic $\mathrm{PGI}_{2}$ production. An mPGES-1-specific inhibitor is expected to be a more effective anti-carcinogenic agent than a COX-2-specific inhibitor.

A variable-number tandem repeat polymorphism was detected in the promoter region of human PGIS gene that is associated with promoter activity ${ }^{32}$. Poole et al. reported that the PGIS promoter polymorphism might affect the risk of the development of colorectal polyps ${ }^{33}$. They showed that having fewer than six repeats on both PGIS alleles was associated with an increased risk of adenoma development compared with the WT genotype, although having more than six repeats reduced the risk. The PGIS promoter polymorphism may be a predictive marker for the risk of colorectal polyps. It is also noteworthy that IP deficiency did not affect the AOM-induced colonic ACF formation ${ }^{34}$. These results suggested that $\mathrm{PGI}_{2}$ might suppress colon carcinogenesis through an unknown receptor other than IP.

A candidate target for the anti-carcinogenic action of $\mathrm{PGI}_{2}$ is peroxisome proliferator-activated receptor $\delta(\operatorname{PPAR} \delta)$. Gupta et al. showed that endogenously synthesized $\mathrm{PGI}_{2}$ could serve as a ligand for PPAR $\delta^{35}$. It was also reported that PPAR $\delta$ deficiency enhanced AOM-induced polyp formation ${ }^{36}$. PGIS-derived $\mathrm{PGI}_{2}$ might act on PPAR $\delta$ and then suppress carcinogenesis. On the other hand, Zuo et al. reported that PPAR $\delta$ deficiency conversely inhibited colon tumorigenesis in mice ${ }^{37}$. Further studies are needed to clarify the involvement of $\mathrm{PGI}_{2} / \mathrm{PPAR} \delta$ in colon carcinogenesis.

In conclusion, our present findings demonstrate that PGIS promotes inflammatory reactions cooperatively with mPGES-1 but attenuates carcinogenesis, in contrast to mPGES-1. Both PGIS and mPGES-1 functionally couple with COX-2 as their upstream enzymes ${ }^{5,15}$. Toward the development of mPGES1-specific inhibitors as novel NSAIDs without adverse side effects, the involvement of PGIS in target illnesses should be fully elucidated.

\section{Methods}

Animals. All animal experiments were performed in accordance with protocols approved by the Institutional Animal Care and Use Committees of Showa University, in accordance with the Standards Relating to the Care and Management of Experimental Animals in Japan. Balb/c and C57BL/6 mice were purchased from Saitama Experimental Animals Supply Co. (Saitama, Japan). mPGES-1 KO mice and PGIS KO mice on a C57BL/6 $\times 129 / \mathrm{SvJ}$ background were described previously ${ }^{9,28}$. In a colon carcinogenesis model, we used these $\mathrm{KO}$ mice, backcrossed 3 times onto Balb/c background ${ }^{11}$. For the establishment of PGIS/mPGES-1 DKO mice, PGIS(-/-) mice were crossed with mPGES-1(-/-) mice to generate PGIS $(+/-)$ mPGES-1 $(+/-)$ mice, and then we intercrossed these heterozygous mice. We also crossed PGIS $(-/-)$ mPGES-1 $(-/-)$ mice with PGIS $(+/-)$ mPGES-1 $(+/-)$ mice to generate PGIS $(+/-)$ mPGES-1(+/-) (as control mice in this study), PGIS $(-/-)$ mPGES-1(+/-) (as PGIS KO mice), PGIS $(+/-)$ mPGES-1(-/-) (as mPGES-1 KO mice), and PGIS $(-/-)$ mPGES-1(-/-) (as DKO mice). These mice were housed in microisolator cages in a pathogen-free barrier facility. All mice were 6-10 weeks old when used for the described experiments.

Induction of peritonitis and preparation and activation of peritoneal $\mathbf{M \Phi s . ~ T h i o g l y c o l l a t e ~}$ medium (Becton Dickinson, Sparks, MD) $(1 \mathrm{~mL} / 20 \mathrm{~g}$ of body weight) was intraperitoneally injected into mice, and peritoneal exudate cells and fluids were collected on day 2 and 4 by washing the cavity with $8 \mathrm{~mL}$ of PBS as described previously ${ }^{9}$. Cell number was determined by Trypan Blue exclusion. Cytocentrifuge preparations were Giemsa-stained, and cell subsets were identified and counted. For preparation of $\mathrm{M} \Phi \mathrm{s}$, the peritoneal cells were washed, counted and then seeded into 12-well plates (Iwaki Glass, Tokyo, Japan) at a cell density of $10^{6} \mathrm{cells} / \mathrm{mL}$ in $1 \mathrm{~mL}$ of RPMI medium (Nissui, Tokyo, Japan) supplemented with $10 \%(\mathrm{v} / \mathrm{v})$ fetal calf serum. After incubation for 2 hours in a $\mathrm{CO}_{2}$ incubator, the supernatants and non-adherent cells were removed. More than $90 \%$ of adherent cells were peritoneal M $\Phi$ s. The cells were then incubated with or without $10 \mu \mathrm{g} / \mathrm{mL}$ LPS from Escherichia coli O111:B4 (Sigma, St. Louis, MO) in medium containing $2 \%$ serum for 24 hours. The culture media were taken for measurements of prostanoids.

Acetic acid writhing reaction. The acetic acid writhing reaction was induced in mice by an intraperitoneal injection of $0.9 \%(\mathrm{v} / \mathrm{v})$ acetic acid solution into mice at a dose of $5 \mathrm{~mL} / \mathrm{kg}$, as described previously $^{9,21}$. For the induction of COX-2, LPS $(10 \mu \mathrm{g} / 0.1 \mathrm{~mL}$ of saline/mouse) was given intraperitoneally 18 hours before the injection of acetic acid solution. The number of writhing responses was counted every $5 \mathrm{~min}$. For the measurement of prostanoids, mice were sacrificed $10 \mathrm{~min}$ after the acetic acid injection, and their peritoneal cavities were washed with $5 \mathrm{~mL}$ of PBS. 
Induction of colonic tumors by AOM treatment. Mice were intraperitoneally injected with AOM at a dose of $10 \mathrm{mg} / \mathrm{kg}$ body weight once a week for 6 weeks as described previously ${ }^{11}$, and then killed 6 or 20 weeks after the last injection of AOM. After laparotomy, the entire colons were dissected and then macroscopically divided into normal-appearing tissues and polyps as normal and tumor tissues, respectively. For the microscopic analysis, the dissected colons were filled with $10 \%$ neutral-buffered formalin and then opened longitudinally from the anus to the cecum. For the analysis of ACF formation, mice were killed 6 weeks after the last injection of AOM, and each colon was stained with $0.2 \%$ methylene blue in PBS. Colon tissues were scored under a light microscope for the number of ACFs or polyps per colon.

Immunoblot analysis. Aliquots of samples were subjected to SDS-PAGE using a 10\% gel (for COX-2 and PGIS) or $15 \%$ gel (for mPGES-1) under reducing conditions. The separated proteins were electroblotted onto nitrocellulose membranes (Schleicher \& Schuell, Hahnenstr, Germany) with a semidry blotter (Bio-Rad Laboratories, Hercules, CA). After blocking with 5\% (w/v) skim milk in Tris-buffered saline (TBS) ( $\mathrm{pH} 7.4)$ containing $0.05 \%(\mathrm{v} / \mathrm{v})$ Tween-20 (TBS-Tween), the membranes were probed with the respective antibodies for 1.5 h (1:5000 dilution for anti-COX-2 antibodies (Santa Cruz Biotechnology, Dallas, TX), 1:2500 dilution for anti-mPGES-1 (Cayman Chemical, Ann Arbor, MI), anti-PGIS (Genway Biotech, SanDiego, CA) and anti- $\beta$-actin antibodies (Sigma, St. Louis, MO) in TBS-Tween). They were then incubated with horseradish peroxidase-conjugated anti-rabbit and anti-mouse immunoglobulin $G$ antibodies (1:5000 dilution in TBS-Tween) for $1 \mathrm{~h}$, and visualized with the ECL western blot system (Perkin-Elmer Life Sciences, Boston, MA), as described previously ${ }^{6,9}$.

Histological staining. After surgery, sections of mouse kidneys were placed in phosphate-buffered formaldehyde overnight, then stored in ethanol and embedded in paraffin. Cross-sections were stained with hematoxylin and eosin and Elastica van Gieson.

Immunohisotochemical analysis. Immunohistochemistry of the tissue sections was performed as described previously ${ }^{9}$. Briefly, the tissue sections were incubated for $15 \mathrm{~min}$ with Target Retrieval Solution (DAKO Japan, Kyoto, Japan), incubated for 10 min with $3 \%(\mathrm{v} / \mathrm{v}) \mathrm{H}_{2} \mathrm{O}_{2}$, washed three times with TBS for $5 \mathrm{~min}$ each, incubated with $5 \%(\mathrm{w} / \mathrm{v})$ skim milk for $30 \mathrm{~min}$, washed three times with TBS-Tween for $5 \mathrm{~min}$ each and incubated for $1 \mathrm{~h}$ with anti-PGIS antibody (Genway Biotech, SanDiego, CA) in TBS (1:100 dilution). The sections were then treated with the Envision staining kit (DAKO Japan), followed by counterstaining with hematoxylin.

RT-PCR analysis. Total RNA was extracted from colon mucosa by homogenization in TRIzol reagent. Two micrograms of RNA from each sample were subjected to a reverse transcription (RT) reaction using the High-Capacity cDNA Reverse Transcription Kit (Applied Biosystems, Carlsbad, CA). A SYBR Green-based protocol and real-time PCR detection system (Applied Biosystems) were used to detect mRNA levels. RNA amounts were normalized against the18S rRNA level.

Measurement of prostanoids by liquid chromatography-electrospray ionization-mass spectrometry (LC-ESI-MS). For the extraction of prostanoids from the peritoneal fluids or culture media, an internal standard $\left(50 \mathrm{pg}\right.$ of $\left.\mathrm{PGB}_{2}\right)$ was added to medium $(500 \mu \mathrm{g})$, and then the medium was acidified by the addition of $100 \mu \mathrm{L}$ of $0.2 \%(\mathrm{v} / \mathrm{v})$ formic acid followed by $500 \mu \mathrm{L}$ of ethyl acetate. The samples were mixed and centrifuged at $20,000 \mathrm{~g}$ for $10 \mathrm{~min}$. The organic layer was retrieved and evaporated to dryness with a vacuum evaporator. Samples were resuspended in $100 \mu \mathrm{L}$ of mobile phase A (water/acetonitrile/ formic acid [63:37:0.02, v/v/v]) and injected into a LC-ESI-MS system. For the extraction of prostanoids from the tissues, the snap-frozen tissues were homogenized at $4^{\circ} \mathrm{C}$ in SET buffer using a bead crusher (uT-01, TAITEC, Saitama, Japan), and then the homogenates were centrifuged at $3000 \mathrm{~g}$ for $5 \mathrm{~min}$ at $4{ }^{\circ} \mathrm{C}$. Supernatants were isolated and adjusted to $\mathrm{pH} 3.0$ with $1 \mathrm{M} \mathrm{HCl}$. An internal standard $\left(50 \mathrm{pg}\right.$ of $\left.\mathrm{PGB}_{2}\right)$ was added to the samples, and then the samples were passed through a Sep-Pak C18 cartridge (Waters, Milford, MA). The retained PGs were eluted with $3 \mathrm{~mL}$ of ethyl acetate/methanol $(9: 1[\mathrm{v} / \mathrm{v}])$. The sample solvents were evaporated, and then the PGs were resuspended in $100 \mu \mathrm{L}$ of mobile phase A and injected into the LC-ESI-MS system.

All mass spectrometric analyses were performed using a Prominence HPLC system (Shimadzu, Kyoto, Japan) equipped with a linear ion trap quadrupole mass spectrometer (QTRAP5500, AB Sciex, Framingham, MA, ), as described previously ${ }^{38}$. Briefly, prostanoids were separated by reverse-phase LC on a TSKgel ODS-100 V column $(2.0 \times 150 \mathrm{~mm}$ inner dia, $5-\mu \mathrm{m}$ particle, Tohso, Tokyo, Japan $)$ at a flow rate of $300 \mu \mathrm{L} / \mathrm{min}$ at $30^{\circ} \mathrm{C}$. The column was equilibrated in mobile phase A (water/acetonitrile/formic acid $[63: 37: 0.02, \mathrm{v} / \mathrm{v} / \mathrm{v}])$. Samples $(10 \mu \mathrm{L})$ were injected using a 50- $\mu \mathrm{L}$ injection loop and eluted with a linear gradient from $0 \%$ to $20 \%$ mobile phase B (acetonitrile/isopropanol $[1: 1, \mathrm{v} / \mathrm{v}]$ ) between 0 and $6 \mathrm{~min}$. Mobile phase B was increased to $55 \%$ from 6 to $6.5 \mathrm{~min}$ and held until $10 \mathrm{~min}$. This phase was increased to $100 \%$ from 10 to $12 \mathrm{~min}$ and held until $16 \mathrm{~min}$. Then, from 16 to $17.5 \mathrm{~min}$, the phase was dropped to $0 \%$ and held there until $20 \mathrm{~min}$. Prostanoids were subsequently analyzed using a tandem quadrupole mass spectrometer via multiple-reaction monitoring (MRM) in negative-ion mode. The transitions monitored were m/z: 351/271 for $\mathrm{PGE}_{2}$ and $\mathrm{PGD}_{2}, 353 / 193$ for $\mathrm{PGF}_{2 \alpha}, 369 / 207$ for 6-ketoPGF $\mathrm{P}_{1 \alpha}, 369 / 195$ for 
$\mathrm{TXB}_{2}$ and $333 / 235$ for $\mathrm{PGB}_{2}$. These prostanoids were identified in samples by matching their MRM signal and $\mathrm{LC}$ retention time with those of a pure standard.

Statistics. Data were expressed as mean \pm standard error of the mean (SEM). Data were analyzed by one-way analysis of variance (ANOVA), and then differences among means were analyzed using Tukey-Kramer multiple comparison tests. P-values $<0.05$ were considered statistically significant.

\section{References}

1. Smith, W. L., DeWitt, D. L. \& Garavito, R. M. Cyclooxygenases: structural, cellular, and molecular biology. Annu. Rev. Biochem. 69, 145-182 (2000).

2. Cha, Y. I. \& DuBois R. N. NSAIDs and cancer prevention: targets downstream of COX-2. Annu. Rev. Med. 58, 239-252 (2007).

3. Smith, W. L., Urade, Y. \& Jakobsson, P. J. Enzymes of the cyclooxygenase pathways of prostanoid biosynthesis. Chem. Rev. 111, 5821-5865 (2011).

4. Bresalier, R. S. et al. Cardiovascular events associated with rofecoxib in a colorectal adenoma chemoprevention trial. N. Engl. J. Med. 352, 1092-1102 (2005).

5. Murakami, M. et al. Regulation of prostaglandin $\mathrm{E}_{2}$ biosynthesis by inducible membrane-associated prostaglandin $\mathrm{E}_{2}$ synthase that acts in concert with cyclooxygenase-2. J. Biol. Chem. 275, 32783-32789 (2000).

6. Kamei, D. et al. Potential role of microsomal prostaglandin E synthase-1 in tumorigenesis. J. Bio. Chem. 278, 19396-19405 (2003).

7. Hara, S. et al. Prostaglandin E synthases: understanding their pathophysiological roles through mouse genetic models. Biochimie 92, 651-659 (2010).

8. Samuelsson, B., Morgenstern, R. \& Jakobsson, P. J. Membrane prostaglandin E synthase-1: a novel therapeutic target. Pharmacol. Rev. 59, 207-224 (2007).

9. Kamei, D. et al. Reduced pain hypersensitivity and inflammation in mice lacking microsomal prostaglandin E synthase-1. J. Biol. Chem. 279, 33684-33695 (2004).

10. Kamei, D. et al. Microsomal prostaglandin E synthase-1 in both cancer cells and hosts contributes to tumour growth, invasion and metastasis. Biochem. J. 425, 361-371 (2010).

11. Sasaki, Y. et al. Microsomal prostaglandin E synthase-1 is involved in multiple steps of colon carcinogenesis. Oncogene 31, 2943-2952 (2012).

12. Xu, D. et al. MF63 [2-(6-chloro-1H-phenanthro[9,10- $d$ ]imidazol-2-yl)-isophthalonitrile], a selective microsomal prostaglandin E synthase-1 inhibitor, relieves pyresis and pain in preclinical models of inflammation. J. Pharmacol. Exp. Ther. 326, 754-763 (2008).

13. Arhancet, G. B. et al. Discovery and SAR of PF-4693627, a potent, selective and orally bioavailable mPGES-1 inhibitor for the potential treatment of inflammation. Bioorg. Med. Chem. Lett. 23, 1114-1119 (2013).

14. Leclerc, P. et al. Characterization of a human and murine mPGES-1 inhibitor and comparison to mPGES-1 genetic deletion in mouse models of inflammation. Prostaglandins Other Lipid Mediat. 107, 26-34 (2013).

15. Ueno, N. et al. Coupling between cyclooxygenase, terminal prostanoid synthase, and phospholipase $\mathrm{A}_{2}$. J. Biol. Chem. 276, 34918-34927 (2001).

16. Boulet, L. et al. Deletion of microsomal prostaglandin $\mathrm{E}_{2}\left(\mathrm{PGE}_{2}\right)$ synthase-1 reduces inducible and basal $\mathrm{PGE}_{2}$ production and alters the gastric prostanoid profile. J. Biol. Chem. 279, 23229-23237 (2004).

17. Hara, S. et al. Isolation and molecular cloning of prostacyclin synthase from bovine endothelial cells. J. Biol. Chem. 269, 19897-19903 (1994).

18. Miyata, A. et al. Molecular cloning and expression of human prostacyclin synthase. Biochem. Biophys. Res. Commun. 200, 1728-1734 (1994).

19. Tone, Y. et al. The regional distribution and cellular localization of mRNA encoding rat prostacyclin synthase. Eur. J. Cell. Biol. 72, 268-277 (1997).

20. Kuwamoto, S., Inoue, H., Tone, Y., Izumi, Y. \& Tanabe, T. Inverse gene expression of prostacyclin and thromboxane synthases in resident and activated peritoneal macrophages. FEBS Lett. 409, 242-246 (1997).

21. Murata, T. et al. Altered pain perception and inflammatory response in mice lacking prostacyclin receptor. Nature 388, 678-682 (1997).

22. Yuhki, K. et al. Prostaglandin receptors $\mathrm{EP}_{2}, \mathrm{EP}_{3}$, and IP mediate exudate formation in carrageenin-induced mouse pleurisy. J. Pharmacol. Exp. Ther. 311, 1218-1224 (2004).

23. Honda, T., Segi-Nishida, E., Miyachi, Y. \& Narumiya, S. Prostacyclin-IP signaling and prostaglandin $\mathrm{E}_{2}-\mathrm{EP}_{2} / \mathrm{EP}_{4}$ signaling both mediate joint inflammation in mouse collagen-induced arthritis. J. Exp. Med. 203, 325-335 (2006).

24. Yuhki, K. et al. Prostaglandin $\mathrm{I}_{2}$ plays a key role in zymosan-induced mouse pleurisy. J. Pharmacol. Exp. Ther. 325, 601-609 (2008).

25. Idborg, H., Olsson, P., Leclerc, P., Raouf, J. \& Jakobsson, P. J. Effects of mPGES-1 deletion on eicosanoid and fatty acid profiles in mice. Prostaglandins Other Lipid Mediat. 107, 18-25 (2013).

26. Trebino, C. E. et al. Redirection of eicosanoid metabolism in mPGES-1-deficient macrophages. J. Biol. Chem. 280, 16579-16585 (2005).

27. Lazarus, M. et al. Immunohistochemical localization of microsomal PGE synthase-1 and cyclooxygenases in male mouse reproductive organs. Endocrinology 143, 2410-2419 (2002).

28. Yokoyama, C. et al. Prostacyclin-deficient mice develop ischemic renal disorders, including nephrosclerosis and renal infarction. Circulation 106, 2397-2403 (2002).

29. Nambiar, P. R. et al. Preliminary analysis of azoxymethane induced colon tumors inbred mice commonly used as transgenic/ knockout progenitors. Int. J. Oncol. 22, 145-150 (2003).

30. Morham, S. G. et al. Prostaglandin synthase 2 gene disruption causes severe renal pathology in the mouse. Cell 83, $473-482$ (1995).

31. Keith, R. L. et al. Pulmonary prostacyclin synthase overexpression chemoprevents tobacco smoke lung carcinogenesis in mice. Cancer Res. 15, 5897-5904 (2004).

32. Iwai, N. et al. Human prostacyclin synthase gene and hypertension: the Suita Study. Circulation 100, 2231-2236 (1999).

33. Poole, E. M. et al. Prostacyclin synthase and arachidonate 5-lipoxygenase polymorphisms and risk of colorectal polyps. Cancer Epidemiol. Biomarkers Prev. 15, 502-508 (2006).

34. Mutoh, M. et al. Involvement of prostaglandin $\mathrm{E}$ receptor subtype $\mathrm{EP}_{4}$ in colon carcinogenesis. Cancer Res. 62, 28-32 (2002).

35. Gupta, R. A. et al. Prostacyclin-mediated activation of peroxisome proliferator-activated receptor $\delta$ in colorectal cancer. Proc. Natl. Acad. Sci. USA 97, 13275-13280 (2000).

36. Harman, F. S. et al. Peroxisome proliferator-activated receptor- $\delta$ attenuates colon carcinogenesis. Nat. Med. 10, $481-483$ (2004). 
37. Zuo, X. et al. Targeted genetic disruption of peroxisome proliferator-activated receptor- $\delta$ and colonic tumorigenesis. J. Natl. Cancer Inst. 101, 762-767 (2009).

38. Kuwata, H. et al. Role of long-chain acyl-coenzyme A synthetases in the regulation of arachidonic acid metabolism in interleukin $1 \beta$-stimulated rat fibroblasts. Biochim. Biophys. Acta 1841, 44-53 (2014).

\section{Acknowledgements}

We thank Naho Yajima, Kenta Shirai, Moe Murata, Natsuki Tamura, Masaya Takamura and Ai Ikezawa for their technical assistance. This work was supported in part by a Grant-in-Aid for Young Scientists (25860100) and a Grant-in-Aid for Scientific Research (B) (21390036 and 25293033) from the Japan Society for the Promotion of Science, and by a Grant-in-Aid for Scientific Research on Innovative Areas (23116515 and 25116720) and a grant for a Private University High Technology Research Center Project from the Ministry of Education, Sports, Science, Culture and Technology of Japan.

\section{Author Contributions}

Y.S. performed experiments and together with S.H. conceived, designed the study and wrote the manuscripts. S.K., A.K., K.M. and M.A. performed experiments. Y.I. and T.I. performed histopathological experiments. Y.N., H.K. and C.Y. contributed to experimental designs. All authors analyzed the results and approved the final version of the manuscript.

\section{Additional Information}

Supplementary information accompanies this paper at http://www.nature.com/srep

Competing financial interests: The authors declare no competing financial interests.

How to cite this article: Sasaki, Y. et al. Genetic-deletion of Cyclooxygenase-2 Downstream Prostacyclin Synthase Suppresses Inflammation Reactions but Facilitates Carcinogenesis, unlike Deletion of Microsomal Prostaglandin E Synthase-1. Sci. Rep. 5, 17376; doi: 10.1038/srep17376 (2015).

(c) (i) This work is licensed under a Creative Commons Attribution 4.0 International License. The images or other third party material in this article are included in the article's Creative Commons license, unless indicated otherwise in the credit line; if the material is not included under the Creative Commons license, users will need to obtain permission from the license holder to reproduce the material. To view a copy of this license, visit http://creativecommons.org/licenses/by/4.0/ 